\title{
A CUT OF LARGE-SCALE STRUCTURE ACROSS THE COMA SUPERCLUSTER
}

\author{
I.D. KARACHENTSEV and A.I. KOPYLOV \\ Special Astrophysical Observatory \\ of the USSR Academy of Sciences, \\ Stavropolsky Kraj, Zelenchukskaja \\ Nyzhny Arkhyz, 357147, U.S.S.R.
}

SUMMARY. Spectral survey of 245 galaxies with $m_{B} \leqslant 17.5$ in the narrow strip $\left\{+28^{\circ} 20^{\prime}>\mathrm{DEC}>+28^{\circ} 10^{\prime}, 15 \mathrm{~h}^{2} 8^{\mathrm{m}} \mathrm{O}>\mathrm{R} . \mathrm{A}>10 \mathrm{~h} 42^{\mathrm{m}} \mathrm{O}\right\}$ confirms a bubblelike type of the large-scale structure around the coma supercluster, have been founded by Lapparent et al. (1986). A correlation function in the volume has the power index $\gamma=1.5$ and the correlation radius $r_{0}=$ $29 \mathrm{Mpc}(\mathrm{H}=75 \mathrm{Km} / \mathrm{s} \mathrm{Mpc})$. For the sample of 14 voids their mean diameter $\left\langle D_{v}\right\rangle$ equals to $(33 \pm 3) \mathrm{Mpc}$. This value coincides with the correlation scale $r_{0}=32$ Mpc for Abell clusters, and also with the mean length of "God finger" for a rich cluster, $2 / 3 \sigma_{\nu} \mathrm{H}^{-1}=32 \mathrm{Mpc}$. We note a presence of a preferential orientation of voids toward the Coma supercluster, $\left\langle D_{v}\right.$ rad $\rangle /\left\langle D_{v}\right.$ tan $\rangle=1.15 \pm 0.8$, that corresponds to a possible peculiar flow toward/outward Coma with $v_{\text {pec }} 400 \pm 200(\mathrm{~km} / \mathrm{s})$ at a scale 100 Mpc. 
140

\section{INTRODUCTION}

Numerous measurements of redshifts for bright galaxies in the central region of the Coma cluster (Tifft, Gregory, 1976, Gregory, Thompson, 1978, Kent, Gunn, 1982) have revealed an unexpected complexity and variety of its structure. Analogous surveys have demonstrated structural richness in other nearby clusters as well: in Perseus, Hercules, Cancer. A neccessity of extending the survey of Coma to its wider vicinity and measurements of redshifts for fainter galaxies was growing evident. Such an observing program was started by us at the 6- meter telescope in 1982 in cooperation with the Institute of Applied Mathematics (Ya.B. Zel'dovich, A.G. Doroshkevich, S.F. Shandarin) and the Tartu Observatory (J. Einasto, E. Tago, P. Tennes). The initial aims of this program were:

a. Determination of the maximum boundaries to which the Coma supercluster extends. Specification of the virial mass from the density profile and from that of radial velocity dispersion.

b. Search for an extended supercorona of Coma assumed to be populated by emission galaxies of low luminosity (Karachentsev, 1982 ).

c. Study of a large-scale structure (voids, shells) in the volume with a depth $Z \sim 0.1$. Estimation of a typical void amplitude and a characteristic thickness of shells/ filaments.

As different from other surveys like a set of selected squares (Kirshner et. al., 1981) we choosed the field configuration as a long narrow strip. The strip is oriented along the declination circle and passes accross the centres of the Coma cluster. The region of survey

$$
\begin{array}{r}
+28^{\circ} 10^{\prime}<\text { DEC }<+28^{\circ} 20^{\prime} \\
10^{h} 42^{m} .0<\text { R.A. }<15^{h_{28}} \cdot 0
\end{array}
$$

is almost symmetric relative to the centre of Coma $\left(12^{\mathrm{h}} 58^{\mathrm{m}} 45^{\mathrm{s}}\right.$, $+28^{\circ} 15^{\prime 16}$ ") with a litie prolongation towards the Cor. Bor. cluster $\left(15^{\mathrm{h}} 20^{\mathrm{m}} \cdot 0+27^{\circ} 48^{\prime}\right)$. The integral area of the strip makes $S=63.0 \times(1 / 6)^{\circ}=10.5 \mathrm{sq}$. degrees.

II. OBSERVATIONS

We inspected the strip on the POSS prints and selected $\sim 500$ brightest galaxies. Their coordinates and apparent 
magnitudes were measured on the plates obtained with the $40-\mathrm{cm}$ astrograph (field $8^{\circ} \times 8^{\circ}$, scale $100 " / \mathrm{mm}$ ). Photometric calibrations of the measurements were made from the data by Godwin et al. (1983) and the galaxies of the catalogue by Zwicky et al. (19611968). Root-mean square errors of the coordinate and magnitude measurements were $8^{\prime \prime}$ and 0.28 , respectively. Out of the photometry results we left in the final sample $n=350$ galaxies with $m_{B} \leqslant 18^{m}$. The density distribution of the number of galaxies brighter than 18.0 in the strip is presented in Fig. 1 with the histogram. Here the number of galaxies per square degree is averaged over the interval $\triangle \mathrm{RA}=2^{\circ}$ and expressed in logarithmic scale. Analogous number of galaxies from the $Z$ wicky catalogue with $\mathrm{m}_{\mathrm{zw}} \leqslant 15.7$ is marked by vertical lines. The points over the histogram indicate the number of galaxies in the strip with $m_{p g} \leqslant 18.8$ taken from the data of Lick counts (Shane, Wirtanen, 1967). The width of a band according to DEC was chosen in both catalogues equal to $1^{\circ}$. The expected number of galaxies in our strip up to the lick survey limit is about 1300 .

In 1983-1987 we made spectral observations of the Coma strip galaxies using the 6-meter telescope of the SAO USSR AS. Over half of the objects have been observed with the image-tube spectrograph in the prime focus cage, the rest of them - at the 1024-channel TV scanner in the Nasmyth focus under the same spectral resolution $\sim 5 \AA$. By the present moment radial velocities have been determined for 245 galaxies, that corresponds to the completeness of our spectral survey $P=0.70$ up to 18.0 or $P=0.80$ up to 17.5 . Coordinates, apparent magnitudes, angular diameters and radial velocities of the galaxies will be published on the completion of the spectral survey (Karachentsev,Kopylov, 1990).

\section{DISCUSSION}

The results of the radial velocity measurements are given by $\{R A, V\}-p l o t$ in Fig.2. Each galaxy is represented by a point. Four objects lie beyond the boundary $V=30000 \mathrm{~km} / \mathrm{s}$. The dashed line shows the region of the survey made by Lapparent et al. (1986). This survey contains radial velocity data for all 1099 galaxies inside $8^{\mathrm{h}}<\mathrm{RA}<17^{\mathrm{h}}, 26^{\circ} .5<\mathrm{DEC}<32.5$ band with 


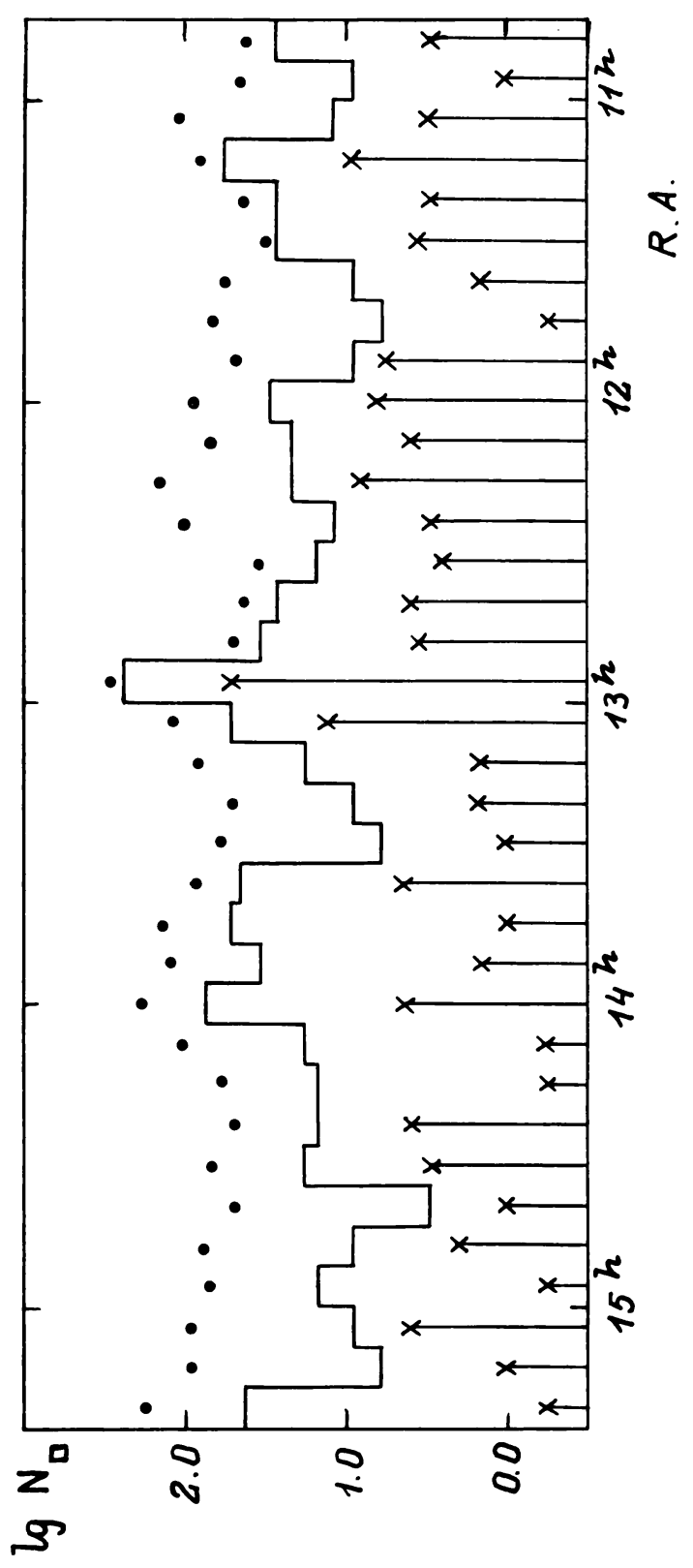

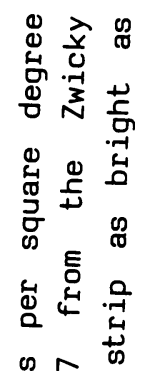

क స

ํㅏํ

元 0 क

4.

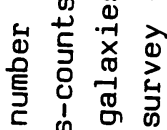

-

ᄂ

ธ્ટّ

苟

出出点空

-1 台品

.

$-10 \begin{array}{ll}0 \\ 0\end{array}$

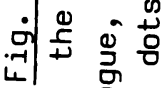

응

栗 


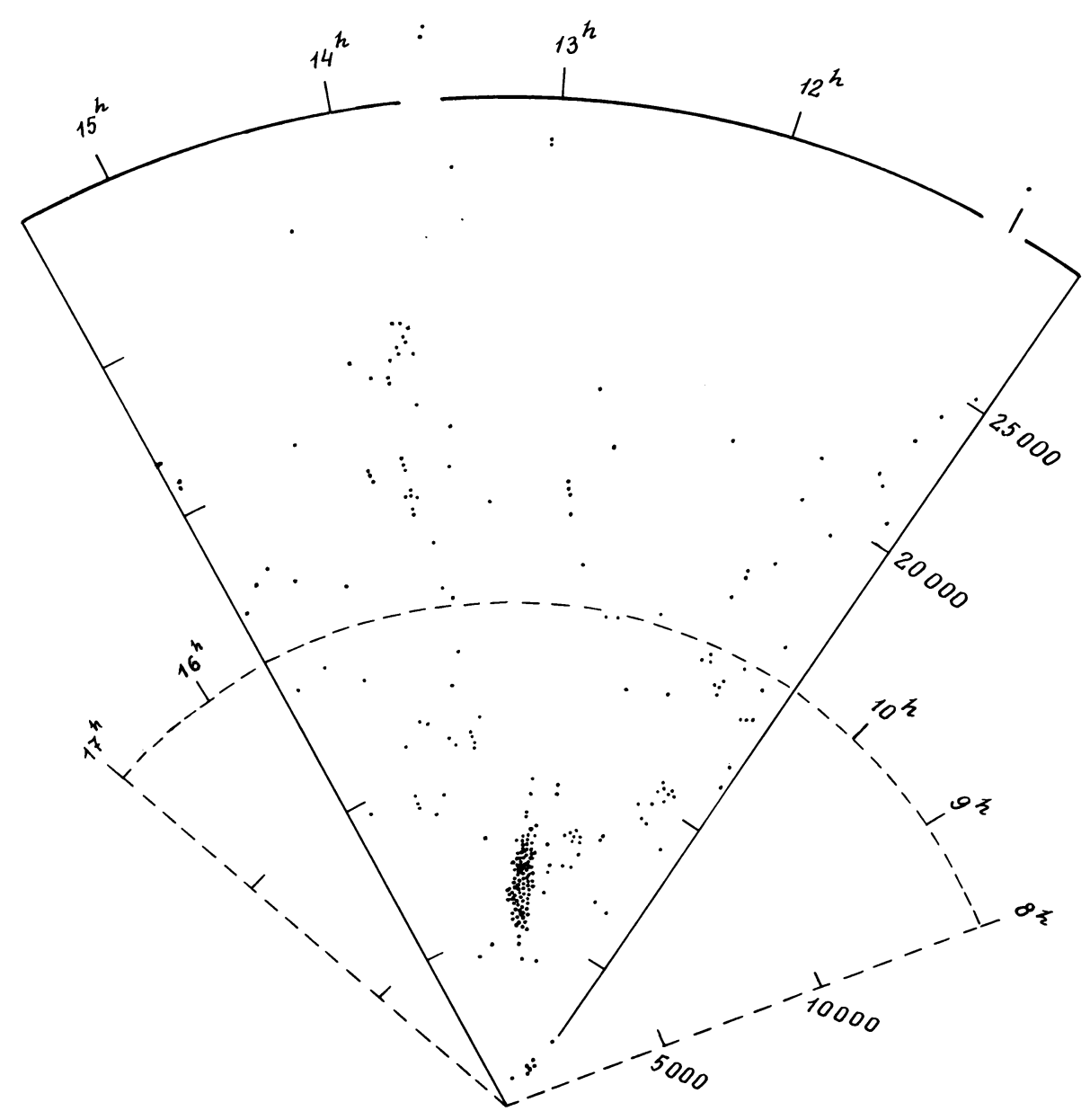

Fig. 2 : Wedge-diagram for distribution of galaxies in the Coma strip according to R.A. and radial velocities $(\mathrm{Km} / \mathrm{s})$. The region surveied by Lapparent et al. (1986) is marked by a dashed line. 
$m_{B}<15^{m} \cdot 5$. We shall address rather often to the data of the survey by Lapparent et al.

The central part of the Coma cluster is distinguished in Fig. 2 by a strong concentration of points stretched along the line of sight. This well known peculiarity is named "God finger" and is caused by the virial motions of galaxies in the cluster. Apparent angular diameter of the Coma in our cut is no higher as the expected Hubble's one, $\Delta R A \simeq 2 \sigma_{V} /\langle v\rangle \simeq 15^{\circ}$, where $\langle V\rangle=6900 \mathrm{~km} / \mathrm{s}$ is the mean velocity of the cluster, and $\sigma_{v}=$ $920 \mathrm{~km} / \mathrm{s}$ is radial velocity dispersion.

In Fig. 2 and analogous diagram by Lapparent et al. (1986) can be seen quite a number of galaxies situated immediately beyond the Hubble's radius of the Coma. However there are no grounds to consider them the members of a regular symmetrical envelope (supercorona) around the Coma cluster. In any case it would be risky to use their coordinates and velocities for the Coma virial mass determination.

To a certain volume a general characteristics of distribution of galaxies is an autocorrelation function $\xi(r)=\mathrm{N}_{g g} / \mathrm{N}_{\mathrm{pp}}-1$. Here $\mathrm{N}_{\mathrm{gg}}(\mathrm{r})$ and $\mathrm{N}_{\mathrm{pp}}(\mathrm{r})$ denote the number of pairs of galaxies and the number of pairs of Poisson's particles simulated in the same volume and in the same quantity as the galaxies. Using for estimation of the spatial distance between two objects in the strip the expression

$$
r^{2}=H^{-2}\left(v_{1}^{2}+v_{2}^{2}-2 v_{1} v_{2} \cos \theta_{12}\right),
$$

$\cos \theta_{12}=\sin D_{1} \cdot \sin D_{2}+\cos D_{1} \cos D_{2}\left(R A_{1}-R A_{2}\right)$

we have calculated the values of $\xi(r)$ for 234 galaxies of the Coma strip with $V<25000 \mathrm{~km} / \mathrm{s}$. The results are presented in Fig. 3 by open circles. Here and below we assume the value of Hubble constant $\mathrm{H}=75 \mathrm{~km} / \mathrm{s} \mathrm{Mpc}$.

Peebles and his cowriters have demonstrated that galaxy distributions in different catalogues can be described by the universal law $\xi(r)=\left(r_{0} / r\right)^{\gamma}$ with the index $\gamma=1.75$ and the correlation radius $r_{0}=(6-7)$ Mpc (Davis, Peebles, 1983). According to Klypin and Kopylov (1983) the same power law, but with $r_{0}=(30-35) \mathrm{Mpc}$, is true to the distribution of rich Abell clusters. During the last time indications have come to appear 
that the values of both parameters, $\gamma$ and $r_{0}$, in the Peebles law are essentially dependant on a type of sample of galaxies. So, from the data by Giovanelli et al. (1986) the $\gamma$ index varies smoothly along the Hubble sequence, running over the values from $\gamma(E)=2.1$ to $\gamma(\mathrm{Sm})=1.3$. For rather faint galaxies in the Coma strip we have had no possibility to determine confidently their structural type. However we found that objects with emission spectra (late types) are characterized by the parameters $\gamma=0.9, r_{0}=11 \mathrm{Mpc}$, while galaxies with absorption spectra have $\gamma=1.5$ and $r_{0}=29 \mathrm{Mpc}$.

Einasto et al. (1986) have provided evidence that the correlation length $r_{0}$ is not the universal constant, but is dependant on a linear size, 1 , of a sample in question. For the most different samples: of galaxies and of systems of galaxies a common statistical relation $r_{0} \sim I^{0.75}$ is detectable. In order to illustrate this scale effect, in Fig.3 we present a correlation function calculated for 33 members of the local group inside the volume $(5.5 \mathrm{Mpc})^{3}$. As can be seen, the parameter $r_{0}$ for galaxies in the Coma strip is one order larger than in the Local group and does not practically differ from the correlation length for Abell clusters.

\section{SOME STEPS TOWARD "VOIDOLOGY" \$)}

Lapparent et al. (1986) have shown that the observed distribution of galaxies in the Coma neighbourhood corresponds best to the picture of bubble- like voids surrounded by surfaces on which galaxies are situated. A typical diameter of a void makes $(30-40) \mathrm{Mpc}$, and a typical thickness of its envelope is $\sim 7 \mathrm{Mpc}$. Such volds can be seen also in our more narrow, but deeper survey. Here for the common space region a good recurrence of "geographic relief" occurs.

\$) "Voidology" is a branch of sience on an edge of art ("bubblegraphy") and religion (dzen). It has no subject to study. The founder - E. Torricelli (1608-1647), who refuted a postulate that nature fears emptiness. Together with Torricelli we claim: "Nature likes emptiness". 


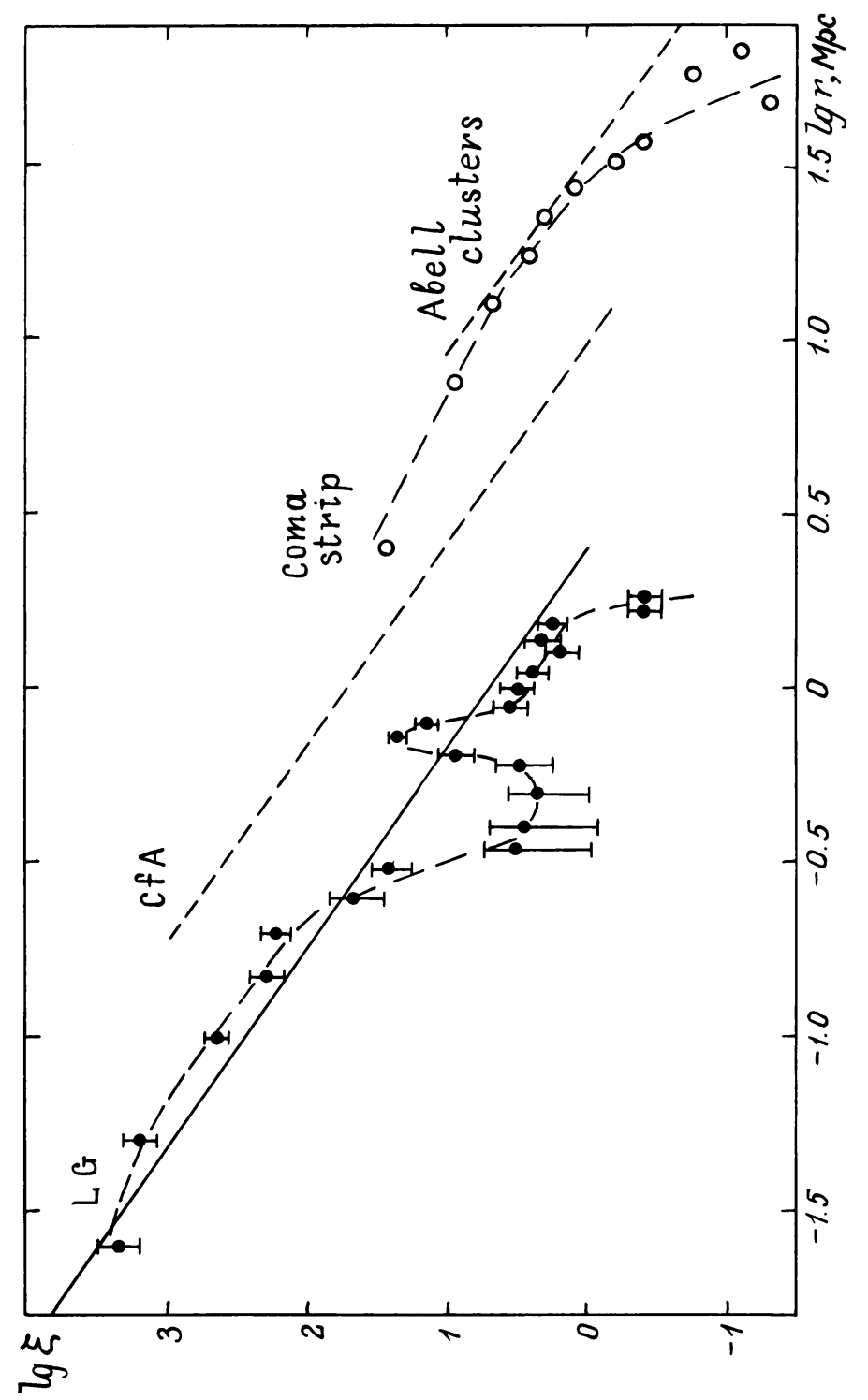

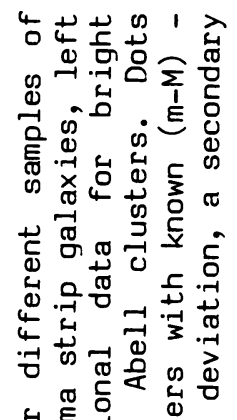

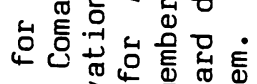

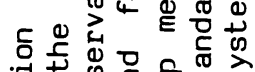

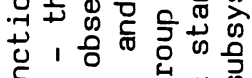
รळ ح出 4 ธิ

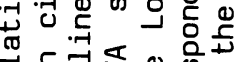
๑ ฐ

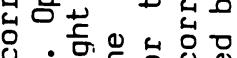

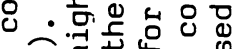
ช0

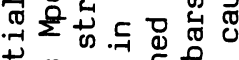
满心

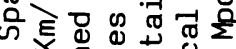

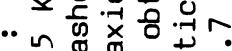
m/

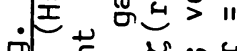

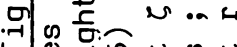

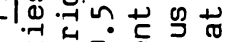

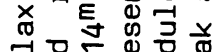

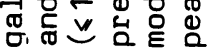


Up to the latest time a discription of individual, the best known empty volumes could be found in literature. We have attempted to have a look at the voids as at a widely spread, ordinary phenomenon and to estimate some mean parameters of voids. For this purpose we have outlined the contours of voids in the common region of the two surveys. To reduce preconception we have posed the task to children, selected common elements of their drawings and smoothed the contours.

A "slice of Swiss cheese" obtained is presented in Fig. 4. 17 "holes" are marked on it, that are numbered in the order of increasing distance from the observer. Naturally, the shape and even the reality of some of the volds can be argued. The subjectivity of plotting the contours is most considerable in the regions, where a statistical completeness of the sample of galaxies is small due to a selection according to their distances. For this ansemble of empty volumes the following parameters and relations are characteristic.

a. A tendency is marked to increasing the diameter of an empty volume with distance from the observer, which is caused by the statistical selection. If three of the remotest objects were excluded, the mean diameter would be $\left\langle D_{v}\right\rangle=\left(\begin{array}{ll}33 & \mp 3\end{array}\right) \mathrm{Mpc}$. Note a remarkable double correspondence: the mean diameter of a void matches the correlation scale for rich clusters, $\left\langle D_{v}\right\rangle \simeq r_{0} \simeq$ 33 Mpc; the radial velocity dispersion in a typical Abell cluster, $\sigma \simeq 700 \mathrm{~km} / \mathrm{s}$, is equivalent to a difference in velocities across a void: $2 \sqrt{3} \sigma_{\mathrm{v}} \mathrm{H}^{-1} \simeq\left\langle\mathrm{D}_{\mathrm{v}}\right\rangle \simeq 32 \mathrm{Mpc}$. Evidently, these numerical fits are not occasional and are directly connected with the origin of the large-scale structure of the Universe.

b. As different from clusters the voids do not show the effect of "God fingers". So, the mean ratio of the radial size to tangential one makes $0.94 \mp 0.08$. Alongside with this a preferential orientation of empty volumes towards the Coma cluster is evident in Fig. 4 (it is marked by a cross). Relative to Coma (but not the observer) the ratio of the radial size of the voids to tangential one is on the average $1.15 \mp 0.08$. Such anisotropy, if it is real, may be indicative of an existence of large-scale peculiar flows with $V_{\text {pec }} \simeq 400 \mathrm{~km} / \mathrm{s}$ called forth by 


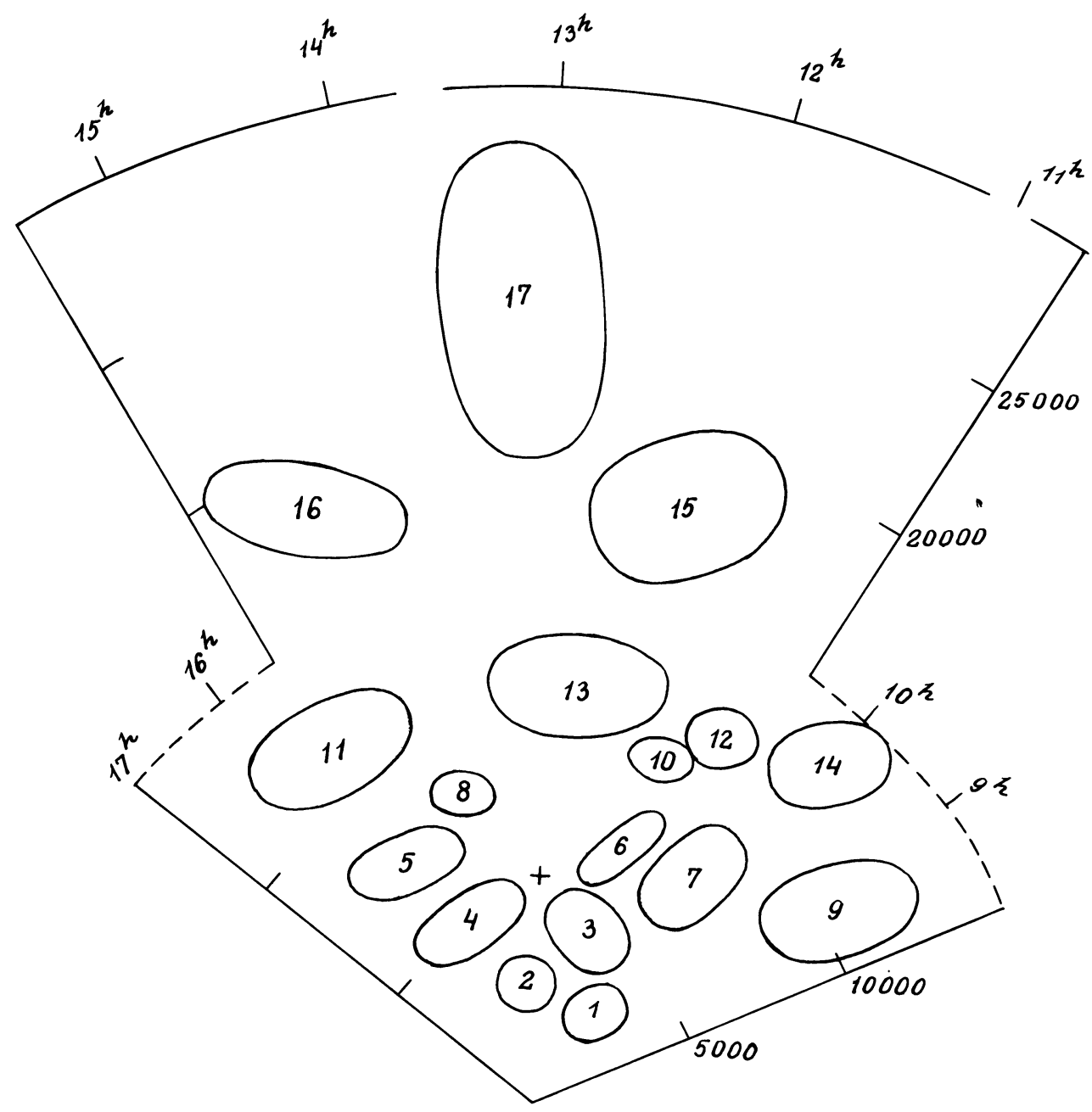

Fig. 4 : Wedge-diagram for distribution of voids according to R.A. and radial velocities $(\mathrm{km} / \mathrm{s})$ in the strip, which passes the Coma cluster center. 
a gravitation field of rich cluster. Some support of this effect can be found on the $\{\mathrm{V}, \mathrm{DEC}\}$ - plots presented by Fairall (1985).

c. In the region $\mathrm{V}<15000 \mathrm{~km} / \mathrm{s}$ the empty volumes were outlined from the distribution of galaxies as bright as 15.5 (Lapparent et al., 1986). If the direct members of the Coma cluster were excluded, only 5 out of 85 galaxies of the fainter survey, having $v<15000 \mathrm{~km} / \mathrm{s}$, would fall inside the marked voids. Almost all of them are near the boundaries of the corresponding voids and could prove to be there as a result of an inaccurate drawing of the contours. With a relative area of voids $s_{v} \simeq 1 / 3$ an assumption that the faint galaxies avoid occasionaly empty regions indicated looks quite unlikely. Note that at a distance of empty volume $\mathrm{n}^{\circ} 2(\mathrm{~V}=3500 \mathrm{~km} / \mathrm{s})$ the absolute magnitude of galaxies $M \simeq-16^{\mathrm{m}}$ corresponds to the limit of our survey $m=17 \cdot 5$. From an indirect estimate the density contrast for the mean void is $\delta \mathrm{n} / \mathrm{n} \leqslant 0.15$ above zero.

\section{FINAI REMARK}

Comparing of the distribution of galaxies in our survey with the data by Lapparent et al. (1986) the essential difference in the width of the two surveys should be kept in mind. At the distance of Coma the strip $\triangle \mathrm{DEC}=10^{\prime}$ corresponds to $0.27 \mathrm{Mpc}$, which is many times smaller than the thickness of a typical bubble envelope. For this reason the question remains open if empty volumes really preserve quite empty under transition from normal luminosity galaxies to dwarfs. To fully answer this question new mass measurements of redshifts for faint galaxies are needed. 
Daris M., Peebles P.J.E., 1983, Astrophys. J., 267, 465. Einasto J., Klypin A.A., Saar E., 1986, Structure of Superclusters and Supercluster Formation. V. Spatial Correlation Function and Voids, Preprint, Tallin.

Fairall A.P., 1985, Redshift Plots of Huchra's Z- Catalogue, Publ. of the University of Cape Town, $n^{0} 8$.

Giovanelli R., Haynes M.P., Chincarini G.L., 1986, Astrophys.J., 300,77 .

Godwin J.G., Metcalfe N., Peach J.V., 1983, MNRAS, 202, 113. Gregory S.A., Thompson I.A., 1978, Astrophys. J., 222, 784. Karachentsev I.D., 1982, Letters to Astron. Zh., 8, 74. Karachentsev I.D., Kopylov A.I., 1990, Astrofizika, in press. Kent S.M., Gunn J.E., 1982, Astron. J., 87, 945.

Kirshner R., Oemler A., Schechter P., Shectman S., 1981, Astrophys. J. Lett., 248, L57.

Klypin A.A., Kopylov A.I., 1983, Letters to Astron. Zh.,9, 75. Lapparent V. de, Geller M.J., Huchra J.P., 1986, Astrophys. J. Lett., 302, I1.

Shane C.D., Wirtanen C.A., 1967, Publication of Lick observ., XXII, part 1 .

Tifft W.G., Gregory S.A., 1976, Astrophys. J., 205, 696. Zwicky F., Herzog E., Wild P., Karpowicz M., Kowal C., 19611968, Catalogue of Galaxies and of Clusters of Galaxies, Paradena, California Institute of Technology. 